\title{
Sensor placement and parameter identifiability in grey-box models of building thermal behaviour
}

\author{
O. M. Brastein*, R. Sharma, N.-O. Skeie \\ Department of Electrical Engineering, Information Technology and Cybernetics \\ University of South-Eastern Norway, N-3918 Porsgrunn, \\ *(ole.m.brastein@usn.no)
}

\begin{abstract}
Building Energy Management systems can reduce energy consumption for space heating in existing buildings, by utilising Model Predictive Control. In such applications, good models of building thermal behaviour is important. A popular method for creating such models is creating Thermal networks, based cognitively on naive physical information about the building thermal behaviour. Such models have lumped parameters which must be calibrated from measured temperatures and weather conditions. Since the parameters are calibrated, it is important to study the identifiability of the parameters, prior to analysing them as physical constants derived from the building structure. By utilising a statistically founded parameter estimation method based on maximising the likelihood function, identifiability analysis can be performed using the Profile Likelihood method. In this paper, the effect of different sensor locations with respect to the buildings physical properties is studied by utilising likelihood profiles for identifiability analysis. The extended 2D profile likelihood method is used to compute two-dimensional profiles which allows diagnosing parameter inter-dependence, in addition to analysing the identifiability. The $2 \mathrm{D}$ profiles are compared with confidence regions computed based on the Hessian.
\end{abstract}

Keywords: building energy management systems, thermal behavior, parameter estimation, parameter identifiability, Profile Likelihood

\section{Introductions}

\subsection{Background}

A significant portion of the worlds total energy production is consumed by heating and cooling of buildings (Perera et al., 2014). Building Energy Management Systems (BEMS) is therefore an important part of the ongoing effort to reduce anthropogenic $\mathrm{CO}_{2}$ emissions. In particular, Model Predictive Control (MPC) has been shown to reduce energy consumption in buildings by utilizing models to predict the thermal behaviour of a building (Fux et al., 2014; Killian and Kozek, 2016). Hence, the development of models of building thermal behaviour has received considerable interest by the scientific community in recent years.

\subsection{Previous work}

A common approach to the modelling of building thermal behaviour is the use of thermal network models (Berthou et al., 2014; Reynders et al., 2014). These models are often described using electric analogues Resistor-Capacitor (RC) circuits. Based on a naive understanding of the thermodynamics involved, these RC circuits constitute simplified lumped parameter models. Parameters are estimated from measurements of temperature inside the building, weather conditions and input power consumed for space heating. As simplified models based on both physical insight and measurement data, thermal network models constitute a compromise between fully physics based white-box and purely data-driven black-box models. This type of model, often called grey-box models, allows use of prior knowledge of the system while also allowing calibration of parameters to adapt the model to a particular building. This approach offers improved prediction accuracy while also allowing use of prior physical information to be injected into the model (Madsen and Holst, 1995; Bacher and Madsen, 2011; Kristensen et al., 2004).

Since the model structure is designed based on knowledge of a particular building, it is often assumed that the parameters are determined by the physical properties of that building. However, since the parameters are identified from data, this assumption needs to be verified in the context of parameter identifiability (Reynders et al., 2014; Deconinck and Roels, 2017; Ferrero et al., 2006). In particular, testing of practical identifiability (Raue et al., 2009), i.e., if sufficient dynamic information about the underlying system is contained in the calibration data (Ferrero et al., 2006), is of importance.

\subsection{Overview of paper}

Since weather is part of the experimental conditions, and, typically, the acceptable range of indoor temperatures, as well as input heater power, is limited, model calibration must usually be performed on sub-optimal data. One element which, to some degree, is open to experimentation is the location of the sensors. As simplified models, thermal networks reduce large indoor spaces and objects, such as the building envelope, to point nodes in the RC circuit. How these nodes correspond to the physical building is 
determined by the sensor location. In this work, we study different sensor placements in an experimental building to show how sensor location, with respect to the physical properties of the building, affects the dynamic information contained in the data and hence the practical identifiability of model parameters. Parameter identifiability is analysed using the Profile Likelihood method, both in the single parameter dimension and in two parameter dimensions by projecting the profile onto a plane in parameter space. The latter method allows improved insight into the parameter domain, including analysing parameter inter-dependence and the effects of a constrained parameter space.

\section{Theoretical basis}

The framework presented in (Kristensen et al., 2004), named Continuous Time Stochastic Modelling (CTSM), is a statistically well founded approach to parameter estimation. The theoretical basis is briefly summarised below. For a more detailed discussion see (Kristensen et al., 2004). Consider the estimation problem:

$$
\begin{aligned}
& \hat{\theta}=\arg \min _{\theta} g(\theta ; \mathscr{M}, \mathscr{K}, \mathscr{A}) \\
& \text { s.t. } \theta \in \Theta
\end{aligned}
$$

Here, $\mathscr{M}$ is a predetermined model structure parametrised by $\theta \in \Theta$, where $\Theta \subseteq \mathbb{R}^{n_{\theta}}$ is a set of feasible values for the model parameters that form inequality constraints for the optimisation problem in Eq. (1). Parameters in $\theta$ are varied over the feasible set $\Theta$ by a numerical optimisation algorithm $\mathscr{A}$. The experimental conditions $\mathscr{K}$ include measurements for the continuous time input $u_{t} \in \mathbb{R}^{n_{u}}$ and output $y_{t} \in \mathbb{R}^{n_{y}}$. The corresponding ordered sequences of discrete time measurements $u_{k}$ and $y_{k}$ taken from the system $\mathscr{S}$ are $y_{[N]}=\left[y_{0}, y_{1}, \ldots, y_{N}\right]$ and $u_{[N]}=\left[u_{0}, u_{1}, \ldots, u_{N}\right]$, where the integer subscripts $k=0,1, \ldots, N$ denote the discrete time sampling instants, and the subscript enclosed in [.] is used to indicate an ordered sequence.

The likelihood function, i.e., the probability of observing the measurement sequence $y_{[N]}$ when $\theta$ and $\mathscr{M}$ are given, is defined:

$$
L\left(\theta ; y_{[N]}, \mathscr{M}\right)=p\left(y_{[N]} \mid \theta, \mathscr{M}\right)
$$

By assuming that the residuals are Normal distributed, and applying the product rule to expand the probability in Eq. (2), we obtain (Kristensen et al., 2004):

$$
L\left(\theta ; y_{[N]}\right)=\left(\prod_{k=1}^{N} \frac{\exp \left(-\frac{1}{2} \varepsilon_{k}^{T} \mathscr{E}_{k \mid k-1}^{-1} \varepsilon_{k}\right)}{\sqrt{\operatorname{det}\left(\mathscr{E}_{k \mid k-1}\right)}(\sqrt{2 \pi})^{n_{y}}}\right) p\left(y_{0} \mid \theta\right)
$$

The quantities $\hat{y}_{k \mid k-1}, \varepsilon_{k}$ and $\mathscr{E}_{k \mid k-1}$, which can be obtained using a Kalman Filter (KF) (Kristensen et al., 2004), is needed for evaluation of the multivariate Gaussian in Eq. (3). By taking the negative logarithm, and eliminating the factor $\frac{1}{2}$, the result $\ell(\theta)=-\ln L(\theta)$, where dependency on $y_{[N]}$ and $\mathscr{M}$ is omitted for simplicity, can be used as the objective $g$ in Eq. (1).

\subsection{Profile likelihood}

Since the model structure $\mathscr{M}$ is a representation of a system $\mathscr{S}$, it is often assumed that $\mathscr{S} \in \mathscr{M}(\Theta)$ and that consequently there exists a true parameter vector $\theta^{*}$ such that $\mathscr{M}\left(\theta^{*}\right)=\mathscr{S}$. However, this is rarely the case, especially for simplified grey-box models based on a naive physical understanding of the system $\mathscr{S}$. Typically, the estimate $\hat{\theta}$ depends on several factors, such as the amount of dynamic information in $\mathscr{K}$, the choice of objective function $g$, and to some extent on the optimisation algorithm $\mathscr{A}$. Hence, prior to interpretation of parameters as physical constants of $\mathscr{S}$, it is necessary to perform an identifiability analysis. Since the parameters are estimated using the Likelihood function, the Profile Likelihood (PL) method (Raue et al., 2009; Deconinck and Roels, 2017) is a natural choice. The likelihood profile $\ell_{\mathrm{PL}}\left(\theta_{i}\right)$ is defined as the minimum $\log$ likelihood for $\theta_{i}$ when the remaining parameters are freely optimised (Raue et al., 2009; Venzon and Moolgavkar, 1988):

$$
\ell_{\mathrm{PL}}\left(\theta_{i}\right)=\min _{\theta_{j \neq i}} g\left(\theta_{j \neq i} ; \mathscr{M}, \mathscr{K}, \theta_{i}\right)
$$

Values of $\theta_{i}$ must be chosen prior to optimising the remaining $\theta_{j \neq i}$ (Raue et al., 2009). The resulting likelihood profile can be plotted as a function of $\theta_{i}$ and subsequently analysed according to the definitions of structural and practical identifiability for likelihood-based confidence intervals (Deconinck and Roels, 2017). The likelihoodbased confidence interval obtains a confidence region by applying a threshold to the likelihood function (Raue et al., 2009; Venzon and Moolgavkar, 1988). Let

$$
\left\{\theta: \ell(\theta)-\ell(\hat{\theta})<\Delta_{\alpha}\right\} \quad, \quad \Delta_{\alpha}=\chi^{2}\left(\alpha, n_{\mathrm{df}}\right)
$$

where $\hat{\theta}$ is a freely estimated, presumed optimal, parameter vector, and the threshold $\Delta_{\alpha}$ is the $\alpha$ percentile of the $\chi^{2}$-distribution with $n_{\mathrm{df}}$ degrees of freedom.

\section{Profile likelihood in two parameter dimensions}

By freely estimating all but one parameter, the PL method essentially projects the $n_{\theta}$ dimensional space $\Theta$ onto the single parameter $\theta_{i}$. This projection is known to overestimate the width of the likelihood-based confidence interval if there are inter-dependent parameters. A step towards remedying this issue is to modify the PL method to hold out two parameters (PL2) rather than one, i.e.;

$$
\ell_{\mathrm{PL} 2}\left(\theta_{i}, \theta_{j}\right)=\min _{\theta_{k \neq i, j}} g\left(\theta_{k \neq i, j} ; \mathscr{M}, \mathscr{K}, \theta_{i}, \theta_{j}\right)
$$

This projects the parameter space $\Theta$ onto the plane $\Theta_{i, j}=$ $\left(\theta_{i}, \theta_{j}\right)$ s.t. $\theta_{i}, \theta_{j} \in \Theta$. In addition identifiability issues, these profiles can also diagnose parameter interdependence by inspecting the shape of the confidence regions. The resulting two-dimensional profiles can be analysed similarly to the one-dimensional profiles (Raue et al., 2009), using the definition in Eq. (5). These profiles are computed for all possible combinations of parameters. A confidence region in the $\Theta_{i, j}$ plane is obtained by applying the $\Delta_{\alpha}$ threshold. Observe that since $\hat{\theta}$ has $n_{\theta}$ free 


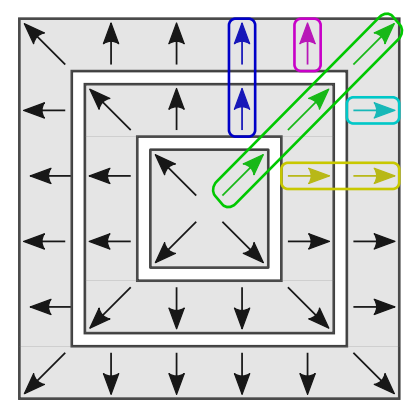

Figure 1. PL2 improved warm start algorithm

parameters while the PL2 estimate has $n_{\theta}-2$, this gives $n_{\mathrm{df}}=2$ for the computation of $\Delta_{\alpha}$ from the $\chi^{2}$-distribution in Eq. (5). The free estimate $\hat{\theta}$ may with advantage be chosen as the minimum $\ell_{\mathrm{PL} 2}\left(\theta_{i}, \theta_{j}\right)$ obtained from all profiles. This search procedure approximates, since it is subject to the brute force discretisation performed in PL2, a free optimisation of all parameters using the already computed $\ell_{\mathrm{PL} 2}$ results. Since the PL2 profiles covers the entire parameter space $\Theta$, this procedure is less affected by local minima than a direct numerical optimisation. Parameter identifiability is obtained if the region is bounded in all directions and the size and shape of this region determines the accuracy of the parameter estimates. If the region contains an unbounded equipotential valley in the log likelihood space, the parameter is considered structurally nonidentifiable. If the profile has a well defined minima, but is unbounded in one direction, i.e., the log likelihood is below the $\Delta_{\alpha}$ threshold, this indicates a practically nonidentifiable parameter (Raue et al., 2009).

\section{Implementation and computation time}

In (Brastein et al., 2019), a brute force method was used, running individual optimisations for each predetermined combination of $\theta_{i}, \theta_{j}$, each iteration starting from the nominal parameter vector $\theta_{0}$. Here, the profiles are constructed by a set of chained optimisations where each new point uses a previously optimised $\hat{\theta}_{k \neq i, j}$ from a near-by point in $\Theta_{i, j}$ as a warm start, working from the centre of the plane $\Theta_{i, j}$ towards the edges. This process is illustrated in Fig. 1. This modification reduced the computation time by approximately 4-10 times, since for each computation of $\ell_{\mathrm{PL} 2}\left(\theta_{i}, \theta_{j}\right)$, the initial guess for the free parameters are taken from a near-by, previously optimised, solution and hence are already close to optimal.

\subsection{Parameter estimation uncertainty}

An estimate of the uncertainty of the estimated parameters can be obtained by computing the covariance of the estimated parameters $\Sigma=2 H^{-1}$ where $H=\left.\nabla^{T} \nabla \ell(\theta)\right|_{\theta=\hat{\theta}}$ is the Hessian of $\ell(\theta)$, whose elements are approximated as (Kristensen et al., 2004; Raue et al., 2009):

$$
\left.h_{i, j} \approx\left(\frac{\partial^{2}}{\partial \theta_{i} \partial \theta_{j}} \ell(\theta)\right)\right|_{\theta=\hat{\theta}}
$$

The partial derivatives of $\ell(\theta)$ can be numerically obtained using the central difference approximation. From the covariance matrix, asymptotic point-wise confidence limits on the estimated parameters can be computed (Raue et al., 2009)

$$
\hat{\theta}_{i} \pm \sqrt{\chi^{2}\left(\alpha, n_{\mathrm{df}}\right) \Sigma_{i, i}}
$$

where for point-wise intervals $n_{\mathrm{df}}=1$. Alternatively, confidence ellipsoids of dimension $n_{\theta}$ as a set of $\theta$ can be defined from the inequality:

$$
\left\{\theta:(\theta-\hat{\theta})^{T} \Sigma^{-1}(\theta-\hat{\theta}) \gtrless \Delta_{\alpha}\right\} \quad, \quad \Delta_{\alpha}=\chi^{2}\left(\alpha, n_{\mathrm{df}}\right)
$$

where the scale of the ellipsoid is determined by the factor $\Delta_{\alpha}$ computed as in Section 2.1 (Johnson and Wichern, 2007). Given that the covariance matrix is symmetric and positive definite, the boundary of an ellipsoid can be obtained by the Cholesky decomposition $\Sigma=L L^{T}$, hence (Press et al., 1992):

$$
(\theta-\hat{\theta})^{T} \Sigma^{-1}(\theta-\hat{\theta})=\Delta \Rightarrow\left|L^{-1}(\theta-\hat{\theta})\right|^{2}=\Delta_{\alpha}
$$

Next, suppose $x$ is a point on a unit hypersphere, then the ellipsoid boundary is obtained by the affine transformation

$$
\theta=\hat{\theta}+\sqrt{\Delta} L x
$$

Elliptic regions in the plane $\Theta_{i, j}$ can be computed by projecting the $n_{\theta}$ dimensional ellipsoid onto $\Theta_{i, j}$. With the $\chi^{2}$ confidence bound given with $n_{\mathrm{df}}=2$, assuming all uncertainty on the parameters in the plane $\Theta_{i, j}$, these elliptic confidence regions are comparable to the PL2 method presented in Section 2.1. Observe that the confidence region based on applying a threshold on the likelihood function as in Eqs. (4) and (6) are often considered superior to the Hessian method, since the Hessian assumes symmetric distributions and therefore cannot be used to identify practical identifiability (Raue et al., 2009). However, the Hessian approach is much faster to compute and gives a reasonable estimate of the estimated parameter uncertainty if the parameters are approximately Normal distributed.

\section{Experimental setup}

\subsection{Experimental building}

The experimental building, which is located at Campus Porsgrunn of the University of South-Eastern Norway (USN), is shown in Fig. 2. The building is constructed with three different types of walls. As shown in Fig. 2, the North wall is constructed using materials with high insulation quality, which is typically used in modern sustainable buildings. The South wall is constructed using traditional building materials, with lower thermal insulation capabilities. 


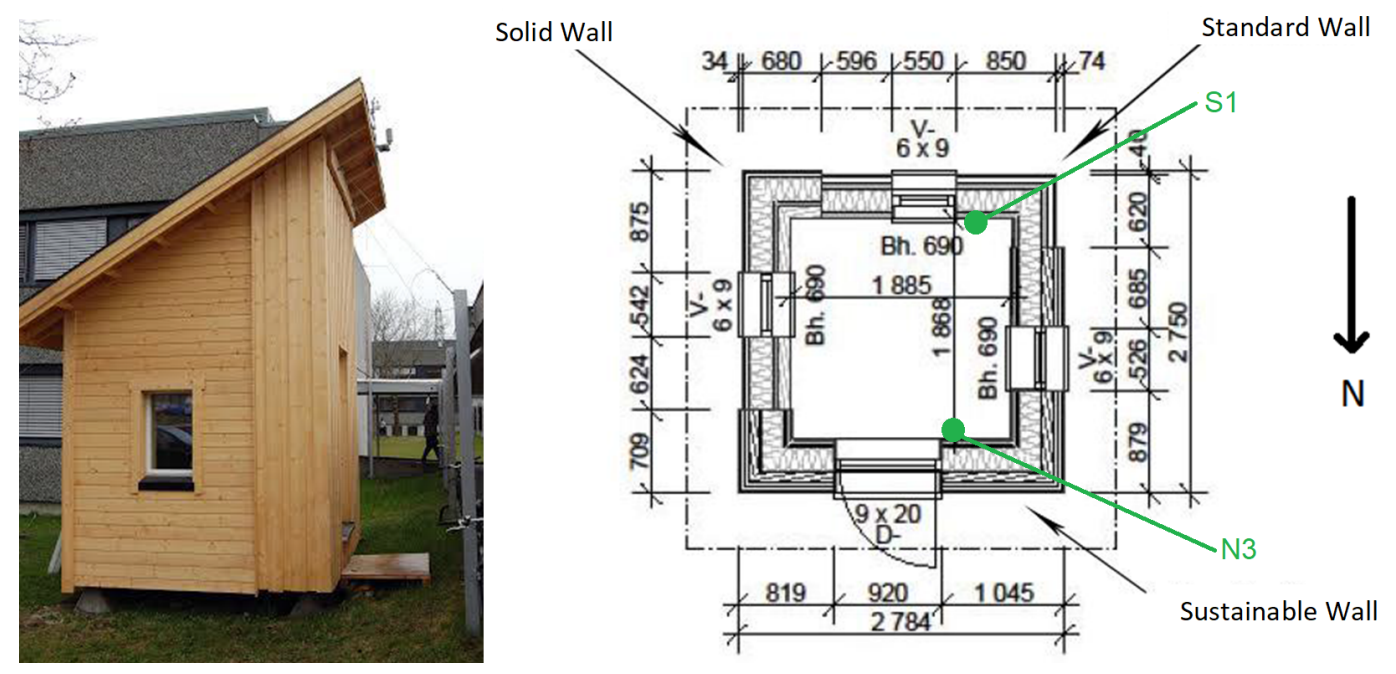

Figure 2. The experimental building has walls constructed using different techniques for insulation. Sensors are located on all walls at different height above floor, and in different insulation layers. The sensors used in this project, N3 and S1, are measuring the wall temperature inside the building on the north and south wall, respectively.

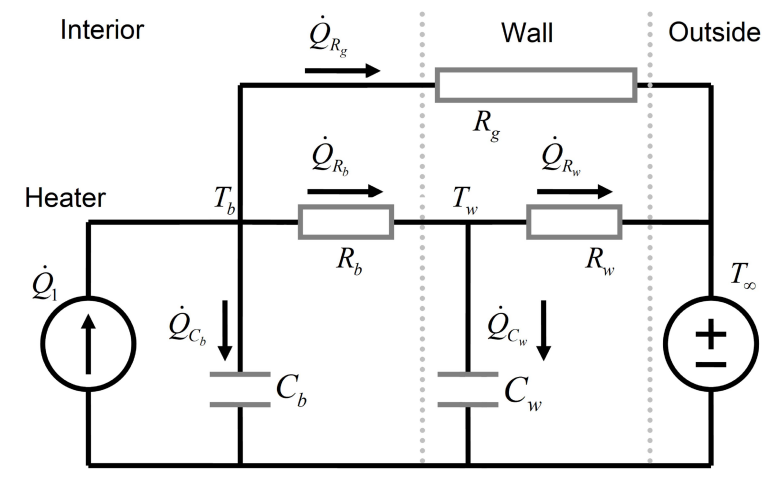

Figure 3. RC circuit model of the building.

\subsection{Model}

Figure 3 shows a possible model structure, which was developed to approximate the thermal behaviour of the experimental building, partially based on the R4C2 model presented in (Berthou et al., 2014). The RC circuit consists of five components: the thermal resistance between room air and wall $R_{\mathrm{b}}$, the building envelope $R_{\mathrm{w}}$, and the thermal resistance of windows and doors $R_{\mathrm{g}}$. The two capacitances $C_{\mathrm{b}}$ and $C_{\mathrm{w}}$ represent the thermal capacitance of the building interior and envelope, respectively. The model has two outputs: the room temperature $T_{\mathrm{b}}$ and the wall surface temperature $T_{\mathrm{w}}$, and two inputs: the consumed power by an electric heating element $\dot{Q}$ and the outside temperature $T_{\infty}$. The parameter vector $\theta$ holds the value of each of the five components. By applying Kirchoff's balance laws to the circuit, the model can be expressed as a linear stochastic differential equation

$$
\begin{aligned}
\frac{d x}{d t} & =A x_{t}+B u_{t}+w_{t} \\
y_{t} & =x_{t}+v_{t}
\end{aligned}
$$

Table 1. Nominal parameter values and $\mathrm{min} / \mathrm{max}$ limits for resistances $[\mathrm{K} / \mathrm{W}]$ and capacitances $[\mathrm{J} / \mathrm{K}]$.

\begin{tabular}{cccccc}
\hline & $R_{\mathrm{g}}$ & $R_{\mathrm{b}}$ & $R_{\mathrm{w}}$ & $C_{\mathrm{b}}$ & $C_{\mathrm{w}}$ \\
\hline$\theta_{0}$ & 0.15 & 0.20 & 0.30 & $1000 k$ & $200 k$ \\
$\theta_{\min }$ & 0.03 & 0.03 & 0.03 & $800 k$ & $1 k$ \\
$\theta_{\max }$ & 0.25 & 2.00 & 2.00 & $1800 k$ & $1000 k$ \\
\hline
\end{tabular}

where

$$
\begin{aligned}
& x_{t}=\left[\begin{array}{c}
T_{b} \\
T_{w}
\end{array}\right], u_{t}=\left[\begin{array}{c}
\dot{Q} \\
T_{\infty}
\end{array}\right], B=\left[\begin{array}{cc}
\frac{1}{C_{b}} & \frac{1}{C_{b} R_{g}} \\
0 & \frac{1}{C_{w} R_{w}}
\end{array}\right] \\
& A=\left[\begin{array}{cc}
-\frac{1}{C_{\mathrm{b}} R_{\mathrm{b}}}-\frac{1}{C_{\mathrm{b}} R_{\mathrm{g}}} & \frac{1}{\mathrm{C}_{\mathrm{b}} R_{\mathrm{b}}} \\
\frac{C_{\mathrm{w}} R_{\mathrm{b}}}{C_{\mathrm{w}} R_{\mathrm{b}}}-\frac{1}{C_{\mathrm{w}} R_{\mathrm{w}}}
\end{array}\right]
\end{aligned}
$$

and $w_{t} \sim N(0, W)$ is the process noise (model error), $W$ is the spectral density of $w_{t}$. All states are measurable, hence Eq. (13) with measurement noise $v_{t} \sim N(0, V)$. Observe that the model equations are expressed in continuous time, and discretised by the estimation software using a RungeKutta 4th (Runge, 1895) order approximation. Observe also that while the model is linear, the algorithm is not restricted to linear models. The choice of Kalman Filter implementation is determined by the type of model being used (Brastein et al., 2019).

Table 1 lists a set of experimentally obtained nominal parameters, which are used as initial guesses for model calibration, and $\mathrm{min} / \mathrm{max}$ limits which corresponds to the bounds of the constrained parameter space $\Theta$.

\subsection{Calibration data}

Figure 4 shows a set of calibration data, which consist of four temperature measurements and one measurement of supplied input power. The data was recorded in February 2018. Originally, the data was collected at 1 minute intervals but has been downsampled to $30 \mathrm{~min}$ time-step, by 


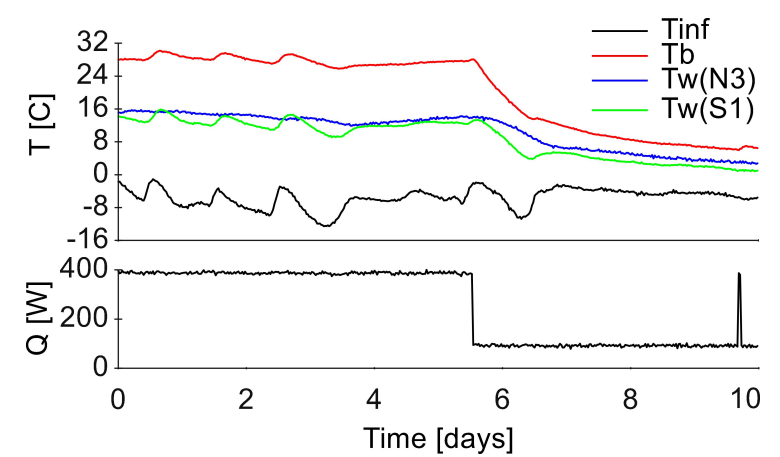

Figure 4. Data recorded from sensor at different locations in the building

Table 2. Estimated covariance matrices with corresponding KS test result (critical value for $95 \%$ conf. is 0.062 ).

\begin{tabular}{c|cccc|cc}
\hline$\#$ & $\mathscr{W}_{T_{\mathrm{b}}}^{\frac{1}{2}}$ & $\mathscr{W}_{T_{\mathrm{w}}}^{\frac{1}{2}}$ & $\mathscr{V}_{T_{\mathrm{b}}}^{\frac{1}{2}}$ & $\mathscr{V}_{T_{\mathrm{w}}}^{\frac{1}{2}}$ & $K S_{T_{\mathrm{b}}}$ & $K S_{T_{\mathrm{w}}}$ \\
\hline S1 & 0.115 & 0.104 & 0.028 & 0.037 & 0.054 & 0.042 \\
N3 & 0.117 & 0.077 & 0.019 & 0.145 & 0.046 & 0.035 \\
\hline
\end{tabular}

extracting every 30th measurement. Two of the temperatures correspond to model state $T_{\mathrm{b}}$ and the model input outdoor temperature $T_{\infty}$. The remaining two measurements correspond to different alternative sensor locations for $T_{\mathrm{w}}$, one on the north wall (sensor N3) and one on the south wall (sensor S1). Figure 4 shows that there is significant differences between these two measurements in dynamic content, due to the different construction materials used in the North and South wall, which will lead to differences in the identifiability analysis of the estimated parameters. In the sequel, two different cases S1 and N3 are analysed, distinguished by the choice of reference measurement for the output $T_{\mathrm{w}}$.

\section{Optimisation algorithm}

In (Brastein et al., 2019) COBYLA (Powell, 1994), based on linear approximations, was used as the optimisation algorithm $\mathscr{A}$ in Eq. (1). In this work, further experimentation with other optimisation algorithms showed that a quadratic approximation algorithm, such as BOBYQA (Powell, 2009), gives significantly faster convergence, by approximately a factor of 5 , as well as more consistent results by improved ability to avoid local minima. BOBYQA is therefore used in the sequel.

\section{Results and discussion}

A requirement for using Kalman Filters to obtain residuals for subsequent evaluation of the likelihood function in Eq. (3) is obtaining reasonable estimates for process and noise covariance matrices, respectively $\mathscr{W}$ and $\mathscr{V}$. In (Brastein et al., 2019) $\mathscr{V}$ was obtained from data, while $\mathscr{W}$ was found by manual experimentation. A better approach is to estimate them from data, by including them in $\theta$.

In order to reduce the number of free parameters, both covariance matrices are assumed diagonal. Further, the

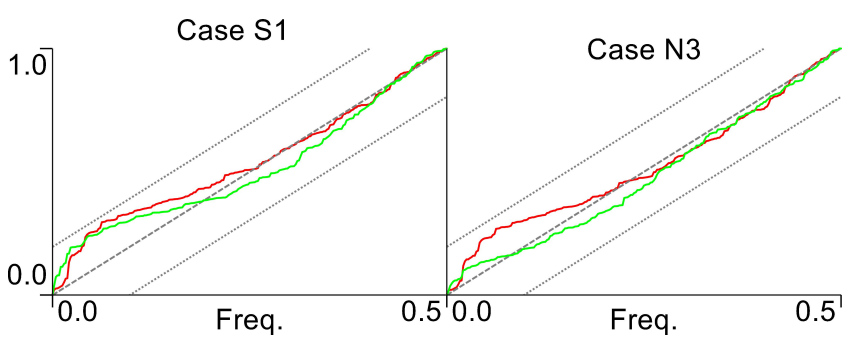

Figure 5. CP diagram of residuals for outputs $T_{\mathrm{b}}(\mathrm{red})$ and $T_{\mathrm{w}}($ green $)$

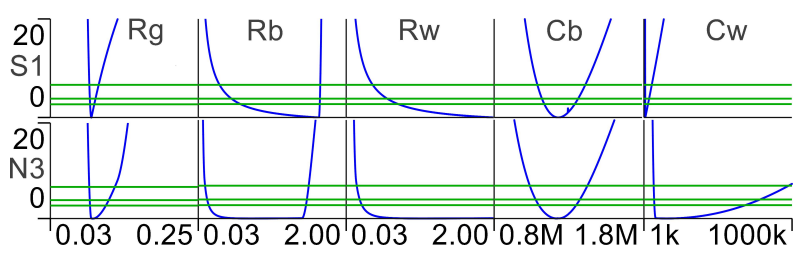

Figure 6. PL results for Cases S1 and N3. Green lines indicate in increasing order $90 \%, 95 \%$ and $99 \%$ confidence limits.

square root of the diagonal elements are added to the parameter vector $\theta$ with some appropriate bounds in $\Theta$ and subsequently estimated by numerical optimisation of Eq. (1). The resulting covariances are shown in Table 2. Observe that the results corresponding to the state/measurement $T_{\mathrm{b}}$ are similar for both cases, while the results for $T_{\mathrm{w}}$ differ significantly. This is expected due to the differences in noise characteristics and dynamic information content in the data collected from the two sensors. For Case $\mathrm{S} 1$ the estimates for $\mathscr{W}$ is similar for both states, where as for Case N3 the differences between measurements of $T_{\mathrm{b}}$ and $T_{\mathrm{w}}$ results in different estimates for the corresponding elements in $\mathscr{W}$.

The residuals obtained after optimising all parameters must be analysed for normality, in order to justify the use of the multivariate Gaussian in Eq. (3) for evaluation of the likelihood function (Kristensen et al., 2004). Figure 5 shows a cumulative periodogram $(\mathrm{CP})$, with $95 \%$ confidence bounds obtained from the Kolmogorov-Smirnov criterion (Madsen, 2007; Madsen and Holst, 1995; Deconinck and Roels, 2017; Bacher and Madsen, 2011). The CP plot shows that the residuals are well approximated by a normal distribution. Additionally, the KolmogorovSmirnov normality-test results are listed in Table 2. After calibration of the parameters, including the noise covariance matrices, residuals are found to pass the normality tests.

\subsection{Profile likelihood}

Once the covariance parameters have been determined, the remainder of this paper is focused on analysing the parameter space $\Theta$ by use of the Profile Likelihood (PL) (Raue et al., 2009) method, first in a single parameter dimension, and next in two parameter dimensions. The PL results in Fig. 6 show, as expected, that some of the parameters have narrower profiles for the S1 Case compared with 


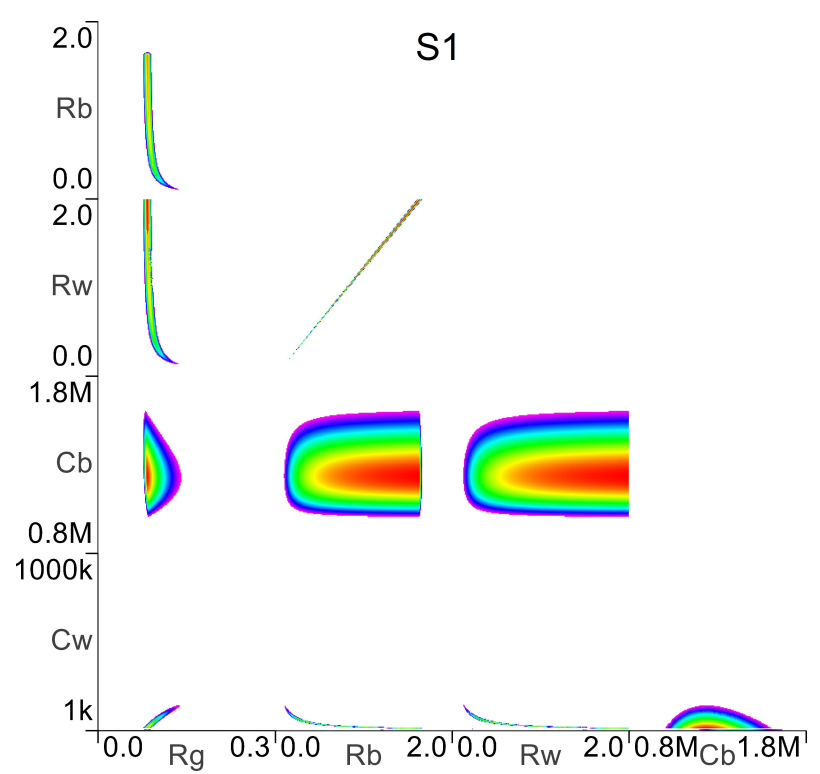

Figure 7. PL2 results for Case S1 (left) and Case N3 (right). Confidence limits, base on the $\chi^{2}$ distribution with 2 degrees of freedom is indicate on the figure legend to the right.

the N3 Case. Since the excitation in $T_{\mathrm{w}}$ is much larger for Case S1, it is expected that the identifiability analysis reflects this by computing tighter confidence regions for the parameters most affected by $T_{\mathrm{w}}$. Observe in particular how the profiles for $C_{\mathrm{w}}$ and $R_{\mathrm{g}}$ indicates considerably improved identifiability of these parameters for Case S1. The profiles for parameter $C_{\mathrm{b}}$ is almost identical, which is expected, since this parameter is not influenced by $T_{\mathrm{w}}$.

A second observation from Fig. 6 is that $R_{\mathrm{w}}$ is diagnosed as practically non-identifiable, since the profile extend towards infinity in the positive direction. Observe also that $R_{\mathrm{b}}$ follows a similar trend, but with an abrupt break in the profile, which leads to a bounded profile for $R_{\mathrm{b}}$. However, if parameters $R_{\mathrm{b}}$ and $R_{\mathrm{w}}$ are interdependent, the projection of the likelihood function for the parameter space $\Theta$ onto $R_{\mathrm{b}}$ will be affected by $R_{\mathrm{w}}$, and subsequently by the constraint imposed by $\Theta$. This type of constraints, in the presence of parameter interdependence, is known to produce such breaks in the computed profiles (Brastein et al., 2019), as discussed in Section 2.1.

\subsection{Profile likelihood in 2D}

Next, the two-dimensional profile likelihood (PL2) method is applied in order to investigate parameter interdependency. The result is shown in Fig. 7. By projecting the ML function in Eq. (3) onto a plane of two parameters, rather then a single parameter axis as in PL, it becomes possible to diagnose parameter identifiability by observing the shape of the confidence regions. First, observe that the PL2 results show a similar improvement in identifiability for Case S1 over Case N3 for the same parameters. The confidence regions for $C_{\mathrm{w}}$ and $R_{\mathrm{g}}$ are significantly reduced for Case $\mathrm{S} 1$, whereas the region for $C_{\mathrm{b}}$ is similar for both cases. Hence, the results of the PL analysis is confirmed

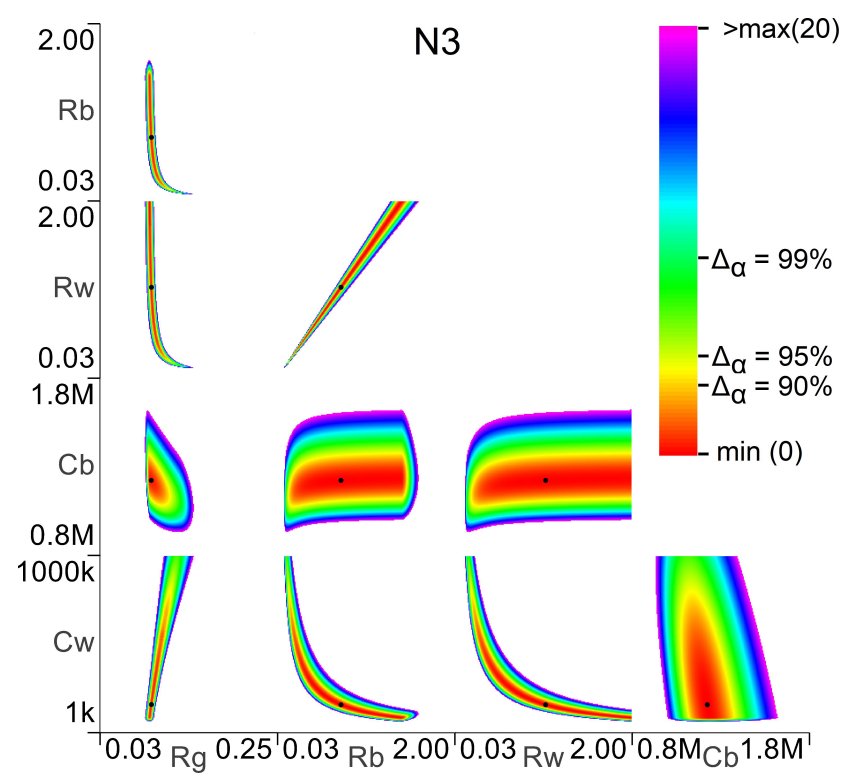

by the PL2 results.

Further, the PL2 results also show that $R_{\mathrm{b}}$ and $R_{w}$ are highly inter-dependent, a fact which was not easily observed in Fig. 6. The projected topology of these two parameters shows a near linear relationship between them. This explains why the PL profile for $R_{\mathrm{b}}$ contains an abrupt break, caused by the constraint on $R_{\mathrm{w}}$ and their interdependence. In this context, it is interesting to consider whether this lack of identifiability for parameters $R_{\mathrm{b}}$ and $R_{\mathrm{w}}$ are of practical, i.e. related to information content in data, or structural nature. Parameter inter-dependency is clearly caused by the model structure, not the data. However, the PL2 profile shows that while the parameters are linearly dependant, e.g. $R_{\mathrm{b}}=c R_{\mathrm{w}}$, neither parameter is identifiable, since the profile is unbounded in one direction in $\Theta$. Hence, it is accurate to claim that these two parameters are practically non-identifiable, but also that there is a structural problem of parameter inter-dependency. The latter may be eliminated by re-parametrising the model, say, by introducing the relationship $R_{\mathrm{b}}=c R_{\mathrm{w}}$ with the constant $c$ pre-determined based on Fig. 7. However, there is no physical reason to assume that the thermal resistance between the inside wall and the building interior should be depending linearly on the thermal resistance of the wall itself, hence this modification of the model equations seems somewhat ad hoc. A better alternative is to modify the RC circuit model structure such that the parameter interdependency is resolved.

\subsection{Reduced order model for Case S1}

From Fig. 6 and 7 for Case $S 1$ it appears that the value of $C_{\mathrm{w}}$ tends towards zero as $R_{\mathrm{b}}$ and $R_{w}$ increases. This could indicate that removing $C_{\mathrm{w}}$, and replacing the state $T_{\mathrm{w}}$ by a measurement $T_{\mathrm{w}}=\frac{T_{\circ} R_{\mathrm{b}}+T_{\mathrm{b}} R_{\mathrm{w}}}{R_{\mathrm{b}}+R_{\mathrm{w}}}$, is an appropriate modification. However, after calibrating the reduced model, the re- 

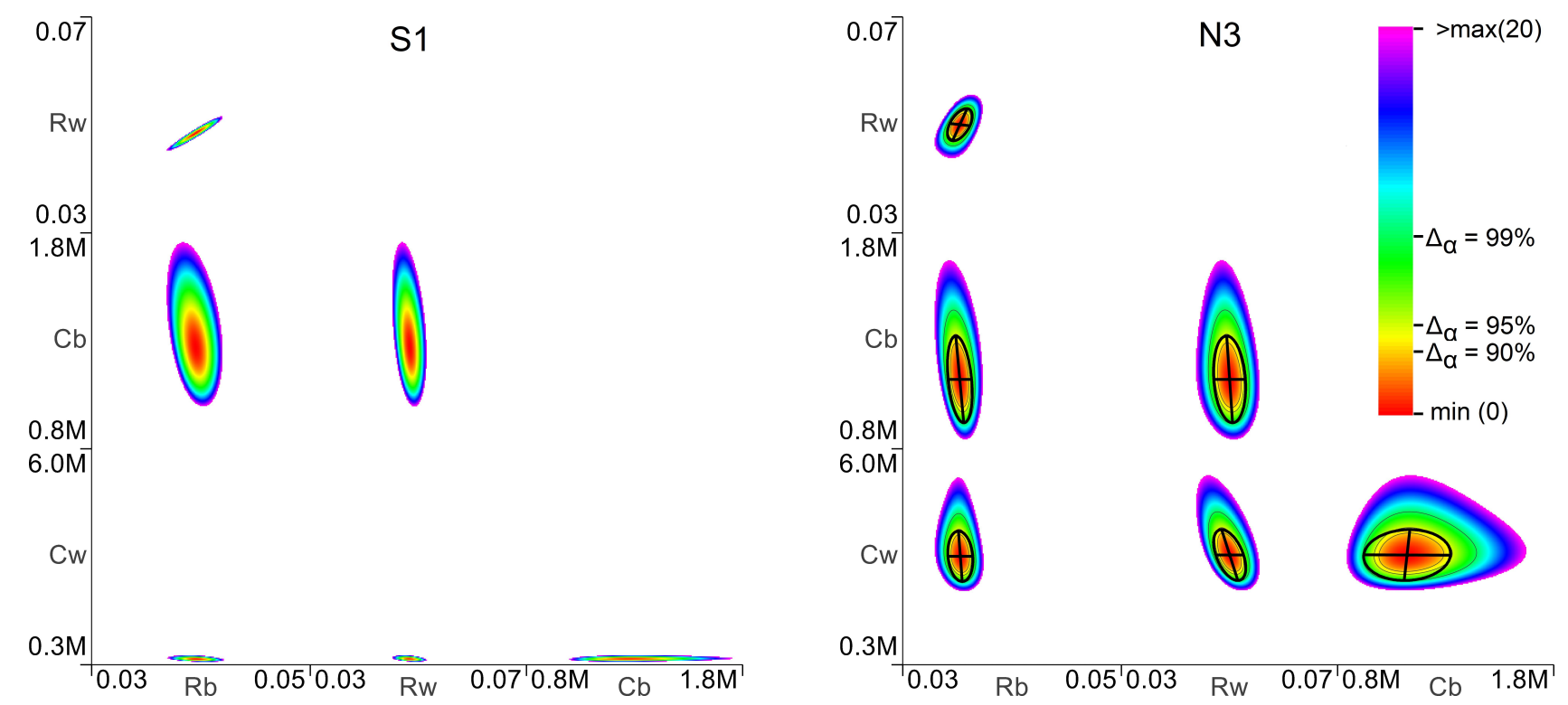

Figure 8. PL2 results after removing $R_{\mathrm{g}}$ from the model, for Case S1 (left) and Case N3 (right). Confidence limits, base on the $\chi^{2}$ distribution with 2 degrees of freedom is indicate on the figure legend to the right. Likelihood threshold isolines and a $95 \%$ confidence error ellipse boundary, based on the inverse Hessian, has been added to the plot for N3.

sidual analysis for output $T_{\mathrm{w}}$, based on a CP diagram, does not support the normality assumption, which in turn indicates that the model order is to low. Hence, removing $C_{\mathrm{w}}$ is not an acceptable modification to the model structure.

\subsection{Parameter inter-dependence}

Observe from Fig. 6 and 7 that for Case N3 there is a significant flat region in the profiles of both $R_{\mathrm{b}}$ and $R_{\mathrm{w}}$. Table 3 contains a selection of values from within this region, which has been computed by keeping $R_{\mathrm{b}}$ constant while optimising the remaining parameters. This experiment shows that by varying the parameters within this optimal region, the total thermal resistance between building interior and the outside temperature, $R_{\mathrm{tot}}=R_{\mathrm{g}} \|\left(R_{\mathrm{b}}+R_{\mathrm{w}}\right)$, where $\|$ indicates a parallel connection of resistors, is constant. Also, the time constant for the wall capacitor $\tau_{\mathrm{w}}=\left(C_{\mathrm{w}} R_{\mathrm{b}} \| R_{\mathrm{w}}\right)$ is approximately constant for the same experiments. Since the total resistance $R_{\mathrm{tot}}<R_{\mathrm{g}}$ it follows that $R_{\mathrm{b}}$ and $R_{\mathrm{w}}$ can grow large, by compensating with $R_{\mathrm{g}}$ and $C_{\mathrm{w}}$, without affecting the models predictions. Combined with the PL2 analysis results, this indicates an overparametrised model.

\subsection{PL analysis of model without Rg}

A natural next step is to reduce the number of parameters by removing the presumed redundant parameter $R_{\mathrm{g}}$ from

Table 3. Optimised values of $R_{\mathrm{w}}, R_{\mathrm{g}}$ and $C_{\mathrm{w}}$ with fixed values of $R_{\mathrm{b}}$ from within the flat region observed in the PL results.

\begin{tabular}{cccccc}
$R_{\mathrm{b}}$ & $R_{\mathrm{W}}$ & $R_{\mathrm{g}}$ & $C_{\mathrm{w}}$ & $R_{\mathrm{tot}}$ & $\tau_{\mathrm{w}}$ \\
\hline 0.700 & 0.993 & 0.093 & $163 k$ & 0.088 & $67.0 k$ \\
0.900 & 1.277 & 0.092 & $126 k$ & 0.088 & $66.5 k$ \\
1.300 & 1.844 & 0.091 & $88 k$ & 0.088 & $67.0 k$ \\
\hline
\end{tabular}

the model circuit. Repeating the PL2 analysis of the model with $R_{\mathrm{g}}$ removed gives the plots shown in Fig. 8. The profiles for all parameter combinations are now approximately elliptical, which indicates parameter independence. Observe also from Fig. 8 that the min/max limits which constitutes the bounds $\Theta$ has been changed to comply with the reduced model structure. Further, all parameters now have bounded profiles, which indicates identifiability. By comparing Case S1 and Case N3, the effect of low excitation in $T_{\mathrm{w}}$ for Case N3 is observed also for the reduced model. In addition to obtaining a different optimal value for $C_{\mathrm{w}}$, as expected, since the sensor is mounted on a different wall, the profile is much wider for Case N3. This indicates a wider confidence region for this parameter, hence a more uncertain estimate.

Table 4. Optimal parameters with $R_{\mathrm{g}}$ removed.

\begin{tabular}{ccccc}
\hline & $R_{\mathrm{b}}$ & $R_{\mathrm{w}}$ & $C_{\mathrm{b}}$ & $C_{\mathrm{w}}$ \\
\hline S1 & 0.040 & 0.048 & $1267 k$ & $419 k$ \\
N3 & 0.035 & 0.051 & $1137 k$ & $2735 k$ \\
\hline
\end{tabular}

With identifiable parameters, it is interesting to compare the optimal parameter estimates, listed in Table 4, for each case. Observe first that for both cases, the total thermal resistance between building interior and outside temperature $R_{\mathrm{b}}+R_{\mathrm{w}} \approx 0.088$, which was the value obtained for the total resistance in Table 3. Observe also that both resistances and the interior capacitance $C_{\mathrm{b}}$ is similar for both cases, while the value obtained for $C_{\mathrm{w}}$ is much larger for the N3 case, as expected, due to the high grade insulation used in the North wall. 


\section{Hessian vs Profile Likelihood}

Observe from Fig. 8 that the super-imposed Hessian based error ellipse at $\alpha=95 \%$ are similar to the $95 \%$ confidence regions computed by thresholds on the likelihood profile. Observe especially for the profile $R_{\mathrm{b}}$ vs $R_{\mathrm{W}}$ that the two confidence region methods produce almost identical regions, since the projected likelihood profile is almost symmetric. For e.g. $C_{\mathrm{b}}$ vs $C_{w}$ the Hessian ellipse and likelihood thresholds are of similar size, but the Hessian has an offset due to the non-symmetric likelihood profile. This shows the advantage of the profile likelihood based regions, in that they can produce accurate results for asymmetric parameter distributions.

\section{Conclusion}

In this paper, two different sensor locations, giving different dynamic information in the recorded calibration data, was used to estimate and analyse parameters of a thermal network grey-box model of building thermal behaviour. The sensor locations differ with respect to the physical properties of the building, with one sensor fitted to a high insulation sustainable wall, and the other to a standard insulation wall. The profile likelihood method was used, projecting the likelihood function in both one and two parameter dimensions, to show the difference in confidence regions produced by lack of excitation in the calibration data. Confidence regions computed by applying a threshold to the 2D profiles were compared with error ellipses computed based on the Hessian, which shows that while the two confidence region methods give similar results, the PL method better represents the uncertainty when the parameter distribution is asymmetric. The twodimensional likelihood profile results were used to diagnose parameter non-identifiability, and the model structure was subsequently modified to resolve the problem, thus obtaining identifiable parameters.

\section{References}

Peder Bacher and Henrik Madsen. Identifying suitable models for the heat dynamics of buildings. Energy and Buildings, 43(7):1511 - 1522, 2011. ISSN 0378-7788. doi:https://doi.org/10.1016/j.enbuild.2011.02.005.

Thomas Berthou, Pascal Stabat, Raphael Salvazet, and Dominique Marchio. Development and validation of a gray box model to predict thermal behavior of occupied office buildings. Energy and Buildings, 74:91-100, 2014.

Ole Magnus Brastein, Bernt Lie, Roshan Sharma, and Nils-Olav Skeie. Parameter estimation for externally simulated thermal network models. Energy and Buildings, 2019. ISSN 03787788. doi:10.1016/j.enbuild.2019.03.018.

An-Heleen Deconinck and Staf Roels. Is stochastic grey-box modelling suited for physical properties estimation of building components from on-site measurements? Journal of Building Physics, 40(5):444-471, 2017.
Cristina Sarmiento Ferrero, Qian Chai, Marta Dueñas Díez, Sverre H Amrani, and Bernt Lie. Systematic analysis of parameter identifiability for improved fitting of a biological wastewater model to experimental data. Modeling, Identification and Control, 27(4):219, 2006.

Samuel F Fux, Araz Ashouri, Michael J Benz, and Lino Guzzella. EKF based self-adaptive thermal model for a passive house. Energy and Buildings, 68:811-817, 2014.

R.A. Johnson and D.W. Wichern. Applied Multivariate Statistical Analysis. Applied Multivariate Statistical Analysis. Pearson Prentice Hall, 2007. ISBN 9780131877153.

M Killian and M Kozek. Ten questions concerning model predictive control for energy efficient buildings. Building and Environment, 105:403-412, 2016.

Niels Rode Kristensen, Henrik Madsen, and Sten Bay Jørgensen. Parameter estimation in stochastic grey-box models. Automatica, 40(2):225-237, 2004.

Henrik Madsen. Time series analysis. Chapman and Hall/CRC, 2007.

Henrik Madsen and Jan Holst. Estimation of continuous-time models for the heat dynamics of a building. Energy and buildings, 22(1):67-79, 1995.

D.W.U. Perera, Carlos F. Pfeiffer, and Nils-Olav Skeie. Modelling the heat dynamics of a residential building unit: Application to Norwegian buildings. Modeling, Identification and Control, 35(1):43-57, 2014. doi:10.4173/mic.2014.1.4.

Michael JD Powell. A direct search optimization method that models the objective and constraint functions by linear interpolation. In Advances in optimization and numerical analysis, pages 51-67. Springer, 1994.

Michael JD Powell. The BOBYQA algorithm for bound constrained optimization without derivatives. Cambridge NA Report NA2009/06, University of Cambridge, Cambridge, pages 26-46, 2009.

William H Press, Saul A Teukolsky, William T Vetterling, and Brian P Flannery. Numerical recipes in $\mathrm{C}++$, volume 2. Cambridge University Press, 1992.

Andreas Raue, Clemens Kreutz, Thomas Maiwald, Julie Bachmann, Marcel Schilling, Ursula Klingmüller, and Jens Timmer. Structural and practical identifiability analysis of partially observed dynamical models by exploiting the profile likelihood. Bioinformatics, 25(15):1923-1929, 2009.

Glenn Reynders, Jan Diriken, and Dirk Saelens. Quality of greybox models and identified parameters as function of the accuracy of input and observation signals. Energy and Buildings, 82:263-274, 2014.

C. Runge. Ueber die numerische Auflösung von Differentialgleichungen. Mathematische Annalen, 46(2):167-178, Jun 1895. ISSN 1432-1807. doi:10.1007/BF01446807.

D. J. Venzon and S. H. Moolgavkar. A method for computing profile-likelihood-based confidence intervals. Journal of the Royal Statistical Society: Series C (Applied Statistics), 37(1): 87-94, 1988. doi:10.2307/2347496. 